\title{
Recurrence patterns in patients with high-grade glioma following temozolomide-based chemoradiotherapy
}

\author{
XIAOFENG ZHOU ${ }^{1}$, XIAOFANG LIAO ${ }^{2}$, BICHENG ZHANG ${ }^{1}$, HUIJUAN HE ${ }^{2}$, \\ YONGJIE SHUI $^{1}$, WENHONG XU ${ }^{1}$, CHAOGEN JIANG $^{3}$, LI SHEN $^{1}$ and QICHUN WEI ${ }^{1}$ \\ ${ }^{1}$ Department of Radiation Oncology, The Second Affiliated Hospital, Zhejiang University School of Medicine, \\ Hangzhou, Zhejiang 310009; Departments of ${ }^{2}$ Radiation Oncology and ${ }^{3}$ Radiology, \\ Quzhou Central Hospital, Quzhou, Zhejiang 324000, P.R. China
}

Received November 15, 2015; Accepted May 20, 2016

DOI: $10.3892 / \mathrm{mco} .2016 .936$

\begin{abstract}
There is currently no consensus regarding the optimal radiation volume for high-grade glioma (HGG). The brain volume irradiated is associated with the extent of radiation neurotoxicity. When reducing the treatment volume, the risk of geographic tumor miss should be considered. In such cases, the recurrence patterns and, particularly, the rate of marginal tumor recurrence, are important indices for determining the optimal radiation volume. In the present study, a smaller-target delineation protocol with limited margins was adopted. The postoperative enhancing tumor and resection cavity were defined as gross tumor volume (GTV); 1 and $2 \mathrm{~cm}$ were added to the GTV to create clinical target volume (CTV1 and CTV2), which received 60 and 54 Gy, respectively. At a median follow-up of 14 months, 54 HGG patients developed tumor recurrence. The median overall and progression-free survival were 14 and 10.5 months, respectively. A total of 34 patients developed central recurrence, 8 presented with in-field recurrence, 2 developed marginal recurrence, 2 had distant recurrence and 11 patients developed cerebrospinal fluid dissemination, 2 of whom developed central recurrence, with 1 patient simultaneously developing marginal recurrence. Local recurrence (central and in-field) was found to be the main recurrence pattern. As the rate of marginal recurrence was low $(<5 \%)$, it appears that the smaller irradiated volume in the present study was appropriate. However, clinical trials investigating limited irradiation volume are required to validate our findings.
\end{abstract}

Correspondence to: Dr Qichun Wei, Department of Radiation Oncology, The Second Affiliated Hospital, Zhejiang University School of Medicine, 88 Jiefang Road, Hangzhou, Zhejiang 310009, P.R. China

E-mail: qichun_wei@zju.edu.cn

Key words: high-grade glioma, recurrence patterns, chemoradiotherapy, irradiated volume

\section{Introduction}

High-grade gliomas [HGG; grade III/IV according to the World Health Organization (WHO) classification] are the most common primary malignant brain tumors in adults, comprising $>50 \%$ of all cases (1). At present, the standard therapy consists of maximal safe surgical resection followed by local brain radiotherapy with concurrent and adjuvant temozolomide (TMZ) chemotherapy $(2,3)$. However, despite aggressive treatment, the median overall survival (OS) of glioblastoma multiforme (GBM) patients after diagnosis is only 14.6 months (2). Recurrence of HGG appears to be unavoidable. The postoperative treatment for HGG was changed from whole-brain radiation therapy (WBRT) plus chemotherapy with carmustine, semustine and dacarbazine (4), to local brain radiotherapy plus TMZ chemotherapy (2). As the irradiated brain volume is associated with radiation neurotoxicity, target delineation is crucial in radiation therapy for glioma.

The Radiation Therapy Oncology Group (RTOG) have provided a protocol for the delineation of the clinical target volume (CTV), including peritumoral edema, for the planning of postoperative radiotherapy for HGG. Historical RTOG protocols, including RTOG 83-02, 86-12 and 97-10, included peritumoral edema in the CTV (5-7). Initial CTV is defined as the gross tumor volume (GTV; postoperative residual tumor and cavity) plus peritumoral edema with an additional $2-\mathrm{cm}$ margin (radiation dose, $46 \mathrm{~Gy}$ ); and boost CTV is defined as the GTV with an additional 2.5-cm margin (radiation dose, $60 \mathrm{~Gy}$ ). The irradiated brain volume is relatively large, according to the RTOG protocol. However, Chang et al (8) argued that CTV delineation using postoperative residual tumor and cavity plus a 2-cm margin, rather than intentionally including peritumoral edema, did not appear to alter the central pattern of failure for patients with GBM. Minniti et al (9) and other European studies $(2,10)$ also concluded that a smaller CTV including the postoperative residual tumor and cavity plus a $2-\mathrm{cm}$ margin, compared with CTV expanded to include the edema, reduced the volume of normal brain tissue subjected to high-dose irradiation, without increasing the risk of marginal recurrence, which was in accordance with the findings of Chang et al (8).

There is currently no consensus regarding target delineation for HGG, whereas the association between smaller 
HGG target delineation and patterns of tumor recurrence has not been extensively investigated in Asian populations. A smaller target delineation protocol with a limited margins was adopted in our institution. In the present study, the patterns of recurrence in 54 HGG patients following TMZ-based chemoradiation were investigated with regards to their correlation with the reduced target delineation protocol.

\section{Patients and methods}

Patients. The clinical data and serial magnetic resonance images (MRI) of 54 patients with recurrent HGG were retrospectively evaluated. Of the 54 patients, 33 were men and 21 were women. The median age was 54 years (range, 22-77 years) and the median Karnofsky performance status was 85 (range, 30-90). A total of 27 patients underwent gross total resection, 25 patients underwent subtotal resection and 2 patients underwent biopsy only. A total of 37 patients were pathologically confirmed as having GBM and the remaining 17 patients as having grade III anaplastic glioma according to the WHO classification (anaplastic astrocytoma, $\mathrm{n}=10$; anaplastic oligodendroglioma, $\mathrm{n}=5$; and anaplastic oligoastrocytoma, n=2) between June 2011 and June 2014. All the patients had received TMZ-based chemoradiation within 2-4 weeks after surgery, followed by adjuvant TMZ chemotherapy. The clinicopathological characteristics of the patients are listed in Table I.

Approval of the present retrospective analysis of the patient data was obtained from the Ethics Committee of the Second Affiliated Hospital of Zhejiang University School of Medicine (Hangzhou, China).

Radiotherapy and chemotherapy. Radiotherapy commenced within 2-4 weeks after surgery. Patients were simulated and treated using a thermoplastic mask for immobilization. The computed tomography (CT) scan obtained at simulation was fused with available post-contrast T1-weighted MRI and the treatment volume was contoured on the fused images. The standard prescribed dose was 60 Gy (2 Gy per daily fraction from Monday to Friday) over a period of 6 weeks. A 6-MV photon beam was delivered by a Varian Trilogy (Varian Medical Systems, Palo Alto, CA, USA) or Siemens Oncor (Siemens AG, Munich, Germany) linear accelerator using a multileaf collimator. According to our protocol, GTV was defined as the resection cavity and any residual contrast-enhancing tumor on post-contrast T1-weighted MRI, ignoring any edematous region. CTV1 was defined as GTV with an added 1-cm margin and CTV2 as GTV with an added $2-\mathrm{cm}$ margin. Peritumoral edema was not intentionally included in the delineation of the CTV, except for any edema existing within the added margin. A 3-mm margin was applied to CTV1 and CTV2 to obtain planning target volume (PTV)1 and PTV2, respectively. For intensity-modulated radiotherapy (IMRT) planning, the dose prescribed for CTV2 was 54 Gy in 30 fractions and the dose for CTV1 was 60 Gy in 30 fractions as a simultaneously integrated boost.

Concomitant chemotherapy consisted of TMZ at $75 \mathrm{mg} / \mathrm{m}^{2} /$ day, administered 7 days per week from the first day of radiotherapy. Adjuvant TMZ chemotherapy was performed for 4 weeks after the end of radiotherapy (administered over 5 days
Table I. Characteristics of patients with recurrent high-grade glioma $(n=54)$.

Characteristics

Patients, n (\%)

\section{Gender}

Male

Female

Type of surgery

Gross total resection

$27(50.0)$

Subtotal resection

Biopsy only

Pathological diagnosis

Glioblastoma

37 (68.5)

Anaplastic astrocytoma

$10(18.5)$

Anaplastic oligodendroglioma

Anaplastic oligoastrocytoma

Age at diagnosis (years)

Median

Range

Karnofsky performance status

Median

Range

$30-90$

every 28 days for $\leq 6$ cycles). The dose of TMZ was $150 \mathrm{mg} / \mathrm{m}^{2}$ for the first cycle and was increased to $200 \mathrm{mg} / \mathrm{m}^{2}$ from the second cycle onwards if the chemotherapy was well-tolerated. The dose was reduced or TMZ was suspended in patients with disease progression or RTOG grade 3-4 toxicity.

MRI examination and assessment of recurrence. Contrast-enhanced MRI of the brain was performed prior to radiotherapy, between the end of radiotherapy and the first cycle of adjuvant TMZ, and every 2 months thereafter or according to the patient's neurological status. The MRI examination protocol included axial T1- and T2-weighted images, as well as axial, sagittal and coronal post-contrast T1-weighted images. According to the current response assessment criteria of the Neuro-Oncology Working Group (11), radiological recurrence was defined as an increase of $25 \%$ or more in the sum of the products of perpendicular diameters of enhancing lesions (compared with baseline if no decrease), or appearance of new lesions. Pseudoprogression in HGG patients often occurred within 2-6 months after the completion of concomitant chemoradiation, disappearing spontaneously within a few months (12-14). Therefore, an asymptomatic increasing lesion around or within the original tumor region, later resolving or regressing, was considered as pseudoprogression $(13,15,16)$, while increasing contrast-enhancing lesions, which continued to progress on serial MRI scans, were defined as tumor recurrence. Recurrence was recorded at the time of the first MRI examination showing tumor progression.

The axial post-contrast T1-weighted images first showing tumor recurrence were imported into the Eclipse Treatment Planning System (Varian Medical Systems) and fused with the planning CT images with associated composite dose distribution. 
Table II. Recurrence patterns and time to recurrence in patients with high-grade glioma.

\begin{tabular}{lccrr}
\hline Recurrence pattern & $\begin{array}{c}\text { Patients, } \\
\text { n }(\%)\end{array}$ & $\begin{array}{c}\text { Time to recurrence } \\
\text { (months), median (range) }\end{array}$ & $\begin{array}{c}\text { Recurrence rate } \\
\text { at 1 year }(\%)\end{array}$ & $\begin{array}{c}\text { Recurrence rate } \\
\text { at 2 years }(\%)\end{array}$ \\
\hline Central recurrence & $34(63.0)$ & $11(2-30)$ & 56 & 94 \\
In-field recurrence & $8(14.8)$ & $9(3-11)$ & 100 & 50 \\
Marginal recurrence & $2(3.7)$ & $30(2-58)$ & 50 & 50 \\
Distant recurrence & $2(3.7)$ & $19(2-35)$ & 55 & 50 \\
CSF-d & $11(20.4)$ & $10(5-58)$ & 50 \\
\hline
\end{tabular}

The time to recurrence was calculated from the date of pathological diagnosis. Of the 11 patients who developed CSF-d, 2 developed central recurrence and 1 simultaneously developed marginal recurrence. CSF-d, cerebrospinal fluid dissemination.

MRI scans obtained immediately prior to and following recurrence were carefully examined to identify the recurrent tumor. The recurrent tumor was contoured and its volume $\left(\mathrm{V}_{\text {recur }}\right)$ was determined based on the CT and fused MRI data sets. $\mathrm{V}_{\text {recur }}$ was delineated without knowledge of the original irradiation volumes, to reduce bias in volume delineation (Fig. 1), and was correlated with the 60-Gy isodose line (IDL) in all patients with recurrence. The recurrence patterns were classified as follows: Central recurrence, $>95 \%$ of the $\mathrm{V}_{\text {recur }}$ inside the 60 -Gy IDL; in-field recurrence, $80-95 \%$ of the $\mathrm{V}_{\text {recur }}$ inside the 60-Gy IDL; marginal recurrence, $20-80 \%$ of the $\mathrm{V}_{\text {recur }}$ inside the $60-\mathrm{Gy}$ IDL; and distant recurrence, $<20 \%$ of the $\mathrm{V}_{\text {recur }}$ inside the $60-\mathrm{Gy}$ IDL $(8,17,18)$. Cerebrospinal fluid dissemination (CSF-d) was considered as another independent recurrence pattern.

Follow-up. The follow-up time was calculated from the date of pathological diagnosis. OS was calculated using the Kaplan-Meier survival analysis and was defined as the time from the day of pathological diagnosis until death or the last follow-up. The time to recurrence was defined from the day of pathological diagnosis until disease recurrence. Statistical analysis was performed with the Statistical Package for Social Sciences, version 19.0 (IBM Corp., Armonk, NY, USA).

\section{Results}

Patient survival. The median follow-up time was 14 months. For GBM cases, the median OS was 13.0 months [95\% confidence interval (CI): 13.4-23.5 months] and the 1-year and 2 -year OS rates were 70.3 and $18.9 \%$, respectively. The median progression-free survival (PFS) was 9.0 months (95\% CI: 7.6-16.6 months) and the 1-year and 2-year PFS rates were 29.7 and $8.1 \%$, respectively.

For anaplastic glioma cases, the median OS was 17.0 months (95\% CI: 13.5-22.2 months) and the 1-year and 2-year OS rates were 82.4 and $17.6 \%$, respectively. The median PFS was 12.0 months (95\% CI: 9.8-17.0 months) and the 1-year and 2-year PFS rates were 47.1 and $11.8 \%$, respectively.

Recurrence patterns. As shown in Table II, 34 patients (63.0\%) developed central recurrence at a median interval of 11 months after pathological confirmation (range, 2-30 months), 8 patients (14.8\%) developed in-field recurrence at a median interval of 9 months (range, 3-11 months), 2 patients (3.7\%) developed marginal recurrence at 2 and 58 months, 2 patients $(3.7 \%)$ developed distant recurrence at 2 and 35 months, and 11 patients $(20.4 \%)$ developed CSF-d at a median interval of 10 months (range, 5-58 months), 2 of whom developed central recurrence, with 1 patient simultaneously developing marginal recurrence. A representative case of GBM recurrence is presented in Fig. 1.

Of the 11 patients who developed CSF-d, 1 patient developed lesions on the leptomeninges of the brainstem, cervical, thoracic and lumbar spine, and a total of 15 lesions were detected in the other 10 cases: In the ependyma of the ipsilateral lateral ventricle $(n=6,40.0 \%)$, in the ependyma of the contralateral lateral ventricle $(n=3,20.0 \%)$, in the lateral cleft pool ( $n=3,20.0 \%$; 1 ipsilateral and 2 contralateral lesions) and in the interpeduncular cistern, fourth ventricle and contralateral pontocerebellar trigone, respectively ( $n=1$ each, 20\%).

\section{Discussion}

A smaller target delineation protocol with a limited margin was used in the present study for TMZ-based chemoradiation therapy in patients with HGG. Local recurrence (central and in-field recurrence) was found to be the major recurrence pattern, while only 2 patients $(3.7 \%)$ developed marginal recurrence.

The volume of the brain subjected to irradiation is considered to be a major factor associated with the development of neurotoxicity, including cognitive decline and radionecrosis. Reduction of the irradiated volume is crucial in HGG radiotherapy, since smaller irradiation volumes may reduce radiation-associated adverse effects. As the prognosis of HGG remains poor, quality of life is an important consideration for patients receiving TMZ-based chemoradiotherapy.

Before the 1980s, postoperative WBRT was used for HGG treatment (19-21). An early study by Wallner et al (22) reported that $\sim 80 \%$ of GBM and anaplastic astrocytoma recurrences after WBRT occurred within $2 \mathrm{~cm}$ of the original pre-operative tumor (22). The results of the Brain Tumor Cooperative Trial 8001 showed that partial brain radiotherapy was as effective as WBRT (23). These important studies provided evidence for the effectiveness of partial-brain radiation therapy for GBM and anaplastic astrocytoma.

To date, there is no consensus regarding the optimal radiation volume for HGG. For example, according to the RTOG guidelines for target delineation, the radiation volume is 

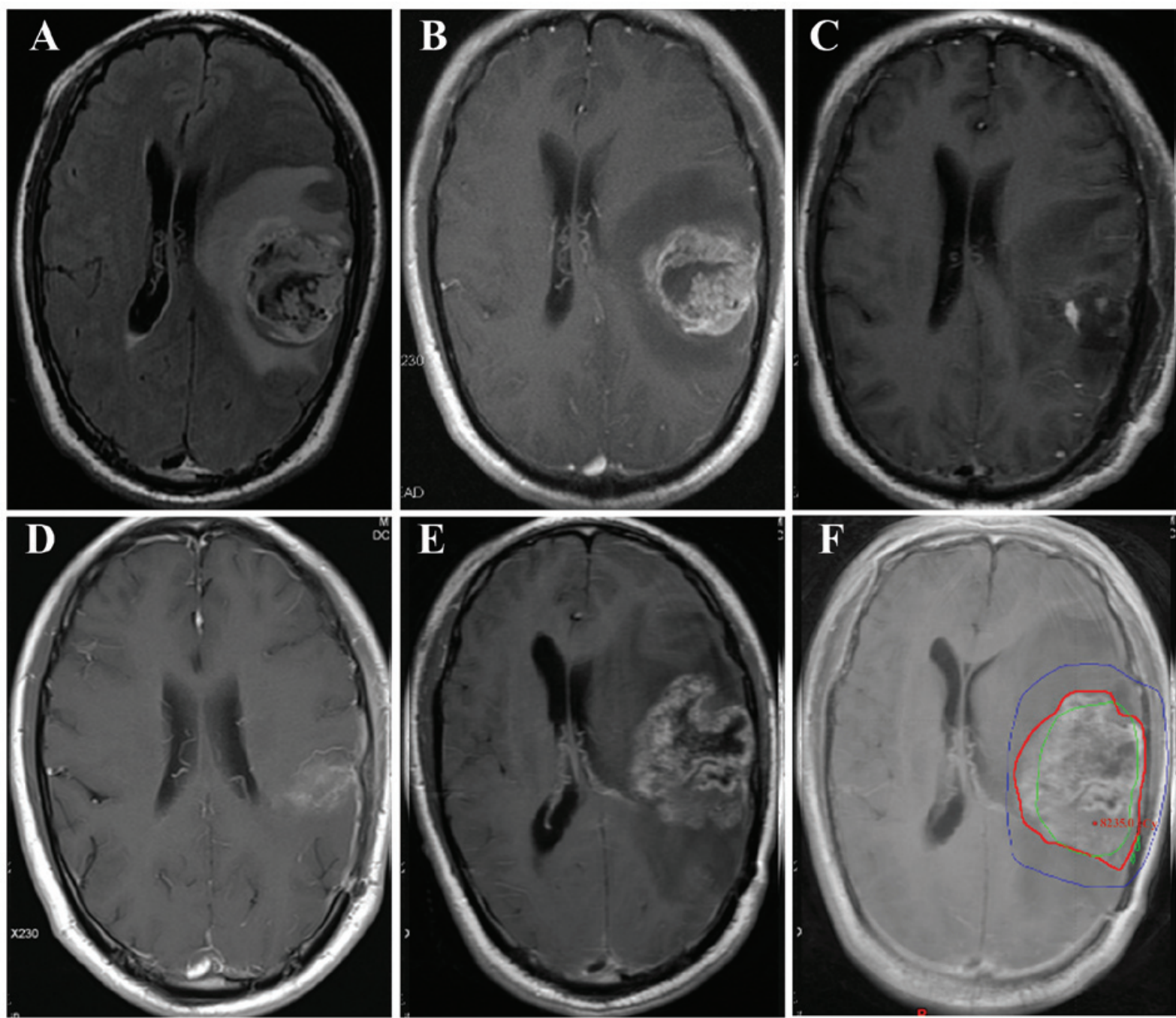

Figure 1. Magnetic resonance images of local glioblastoma multiforme (GBM) recurrence in a representative case. Preoperative (A) axial fluid-attenuated inversion recovery image and (B) post-contrast T1-weighted image showing a tumor located in the left temporal lobe. (C) Postoperative axial post-contrast T1-weighted image. (D) Axial post-contrast T1-weighted image at the end of radiotherapy. (E) Axial post-contrast T1-weighted image at 10 months after pathological diagnosis, showing an increasing enhancing lesion at the primary site. The patient underwent re-operation and had the same pathological diagnosis (GBM) as that of the primary tumor. (F) The axial post-contrast T1-weighted image at recurrence was fused with the planning computed tomography image, revealing local recurrence. The blue line indicates the 54-Gy IDL, the green line indicates the 60-Gy IDL and the red line marks the tumor recurrence volume. IDL, isodose line.

significantly larger compared with that determined following the European Organization for Research and Treatment of Cancer (EORTC) guidelines $(2,10)$. When considering reducing the irradiation volume, the risk of geographic tumor miss should also be considered. In such cases, the rate of marginal tumor recurrence is an important index.

In a study by Milano et al (24), the RTOG guidelines for target delineation were followed: Initial CTV was defined as peritumoral edema with an added margin of $2 \mathrm{~cm}$ and a prescribed radiation dose of 46-50 Gy, whereas boost CTV was defined as the GTV plus a $2-2.5-\mathrm{cm}$ margin, with a prescribed radiation dose of $60 \mathrm{~Gy}$. The irradiated brain volume determined by these guidelines is relatively large. Among the 39 cases with recurrence, as regards the pattern of first recurrence, in-field recurrence occurred in $92 \%$, marginal recurrence in $15 \%$ and distant recurrence in $13 \%$ of the patients. A limited margin was used by McDonald et al (25), who added a median margin of $0.7 \mathrm{~cm}$ to postoperative edema (T2 abnormality) to create the initial CTV, followed by addi- tion of a geometric 0.3 - or 0.5 -cm margin to create the initial PTV. To delineate boost CTV, a $0.5-\mathrm{cm}$ margin was most commonly added to the GTV; boost PTV was calculated by adding a further 0.3 - or $0.5-\mathrm{cm}$ geometric margin, and received a dose of $60 \mathrm{~Gy}$. On average, the treated boost PTV (median, $140 \mathrm{~cm}^{3}$ ) was $70 \%$ smaller compared with the boost PTV with a $2.5-\mathrm{cm}$ margin (median, $477 \mathrm{~cm}^{3}$ ). Of the 41 patients with GBM recurrence, 38 (93\%) had central or in-field recurrence, $2(5 \%)$ had marginal recurrence and $1(2 \%)$ had distant recurrence. A boost PTV margin of $\leq 1 \mathrm{~cm}$ did not appear to increase the risk of marginal recurrence.

Further reduction of radiation volume may be possible for GBM treatment. Chang et al (8) investigated 48 cases of postoperative GBM who were treated at the MD Anderson Cancer Center. GTV was defined as the postoperative enhancing tumor and resection cavity; initial CTV was defined as GTV plus a 2-cm margin, and boost CTV was defined as the GTV plus $0.5 \mathrm{~cm}$. A 5 -mm margin was added to the initial CTV and boost CTV to create the initial PTV and boost PTV, respectively, 
which received 50 and $60 \mathrm{~Gy}$, respectively. A total of 43 patients were found to have central or in-field recurrence, 2 had distant recurrence and $3(6.25 \%)$ had marginal recurrence. The authors concluded that CTV delineation using the postoperative residual tumor and cavity plus a 2-cm margin, rather than intentionally including peritumoral edema, resulted in a smaller brain volume being irradiated compared with the corresponding theoretical RTOG protocol, but did not appear to alter the central pattern of treatment failure for patients with GBM (8).

According to recent EORTC randomized trials, Minniti et al (9) determined the CTV as the residual tumor and resection cavity plus a $2-\mathrm{cm}$ margin. The CTV was expanded by $0.3 \mathrm{~cm}$ to create the PTV to compensate for variability in treatment setup and patient motion, and a dose of 60 Gy was delivered in 30 2-Gy fractions over a period of 6 weeks. For each patient, a theoretical plan based on the addition of postoperative edema plus a $2-\mathrm{cm}$ margin according to the RTOG guidelines was created and the patterns of failure were also evaluated. Central and in-field recurrence was observed in 85 , marginal recurrence in 6 and distant recurrence in 14 patients. The patterns of failure were similar to those previously reported (24); however, the median brain volume subjected to high doses of radiation in the study by Minniti et al (9) was significantly smaller according to the EORTC compared with the RTOG guidelines. The authors concluded that a smaller CTV, including the postoperative residual tumor and cavity plus a 2-cm margin, as compared with CTV expanded to include the edema, reduced the volume of normal brain subjected to high doses of radiation, while not increasing the risk of marginal recurrence.

In the present study, an even smaller CTV, defined as the enhanced residual tumor and cavity plus a $1-\mathrm{cm}$ margin, was introduced as routine treatment and a simultaneously integrated boost IMRT was performed. The recurrence patterns were similar to those reported by Minniti et al (9) and other European studies $(2,10)$, with only 2 patients $(3.7 \%)$ developing marginal recurrence. The median OS and PFS were 13 and 9.0 months, respectively, which were similar to those reported by several prospective studies $(2,9,26)$. As the rate of marginal recurrence was low, it appears that the smaller irradiated volume used in the present study was appropriate. However, clinical trials investigating smaller irradiation volumes in GBM are required to validate our findings.

\section{Acknowledgements}

The authors would like to thank all colleagues at the Department of Radiation Oncology (The Second Affiliated Hospital, Zhejiang University School of Medicine, Hangzhou, China) for their cooperation. Financial support was received from the National Natural Science Foundation of China (grant nos. 81071823 and 81572952).

\section{References}

1. Mirimanoff R-O: High-grade gliomas: Reality and hopes. Chin J Cancer 33: 1-3, 2014.

2. Stupp R, Mason WP, Van Den Bent MJ, Weller M, Fisher B, Taphoorn MJ, Belanger $\mathrm{K}$, Brandes AA, Marosi C, Bogdahn U, et al: Radiotherapy plus concomitant and adjuvant temozolomide for glioblastoma. N Engl J Med 352: 987-996, 2005
3. Stupp R, Hegi ME, Mason WP, van den Bent MJ, Taphoorn MJ, Janzer RC, Ludwin SK, Allgeier A, Fisher B, Belanger K, et al: Effects of radiotherapy with concomitant and adjuvant temozolomide versus radiotherapy alone on survival in glioblastoma in a randomised phase III study: 5-year analysis of the EORTC-NCIC trial. Lancet Oncol 10: 459-466, 2009.

4. Chang C, Horton J, Schoenfeld D, Salazer O, Perez-Tamayo R, Kramer S, Weinstein A, Nelson J and Tsukada Y: Comparison of postoperative radiotherapy and combined postoperative radiotherapy and chemotherapy in the multidisciplinary management of malignant gliomas. A joint Radiation Therapy Oncology Group and Eastern Cooperative Oncology Group study. Cancer 52: 997-1007, 1983.

5. Nelson DF, Curran WJ Jr, Scott C, Nelson JS, Weinstein AS, Ahmad K, Constine LS, Murray K, Powlis WD, Mohiuddin M, et al: Hyperfractionated radiation therapy and bis-chlorethyl nitrosourea in the treatment of malignant glioma - possible advantage observed at $72.0 \mathrm{~Gy}$ in $1.2 \mathrm{~Gy}$ B.I.D. fractions: Report of the Radiation Therapy Oncology Group protocol 8302. Int J Radiat Oncol Biol Phys 25: 193-207, 1993.

6. Urtasun RC, Kinsella TJ, Farnan N, DelRowe JD, Lester SG and Fulton DS: Survival improvement in anaplastic astrocytoma, combining external radiation with halogenated pyrimidines: Final report of RTOG 86-12, phase I-II study. Int J Radiat Oncol Biol Phys 36: 1163-1167, 1996.

7. Colman H, Berkey BA, Maor MH, Groves MD, Schultz CJ, Vermeulen S, Nelson DF, Mehta MP and Yung WK; Radiation Therapy Oncology Group: Phase II Radiation Therapy Oncology Group trial of conventional radiation therapy followed by treatment with recombinant interferon-beta for supratentorial glioblastoma: Results of RTOG 9710. Int J Radiat Oncol Biol Phys 66: 818-824, 2006.

8. Chang EL, Akyurek S, Avalos T, Rebueno N, Spicer C, Garcia J, Famiglietti R, Allen PK, Chao KC, Mahajan A, et al: Evaluation of peritumoral edema in the delineation of radiotherapy clinical target volumes for glioblastoma. Int J Radiat Oncol Biol Phys 68: 144-150, 2007.

9. Minniti G, Amelio D, Amichetti M, Salvati M, Muni R, Bozzao A, Lanzetta G, Scarpino S, Arcella A and Enrici RM: Patterns of failure and comparison of different target volume delineations in patients with glioblastoma treated with conformal radiotherapy plus concomitant and adjuvant temozolomide. Radiother Oncol 97: 377-381, 2010.

10. Brandes AA, Stupp R, Hau P, Lacombe D, Gorlia T, Tosoni A, Mirimanoff RO, Kros JM and van den Bent MJ: EORTC study 26041-22041: Phase I/II study on concomitant and adjuvant temozolomide (TMZ) and radiotherapy (RT) with PTK787/ZK222584 (PTK/ZK) in newly diagnosed glioblastoma. Eur J Cancer 46: 348-354, 2010.

11. Wen PY, Macdonald DR, Reardon DA, Cloughesy TF, Sorensen AG, Galanis E, DeGroot J, Wick W, Gilbert MR, Lassman AB, et al: Updated response assessment criteria for high-grade gliomas: Response assessment in Neuro-Oncology Working Group. J Clin Oncol 28: 1963-1972, 2010.

12. de Wit MC, de Bruin HG, Eijkenboom W, Sillevis Smitt PA and van den Bent MJ: Immediate post-radiotherapy changes in malignant glioma can mimic tumor progression. Neurology 63 : 535-537, 2004.

13. Taal W, Brandsma D, de Bruin HG, Bromberg JE, Swaak-Kragten AT, Smitt PA, van Es CA and van den Bent MJ: Incidence of early pseudo-progression in a cohort of malignant glioma patients treated with chemoirradiation with temozolomide. Cancer 113: 405-410, 2008.

14. Chaskis C, Neyns B, Michotte A, De Ridder M and Everaert H: Pseudoprogression after radiotherapy with concurrent temozolomide for high-grade glioma: Clinical observations and working recommendations. Surg Neurol 72: 423-428, 2009.

15. Brandes AA, Franceschi E, Tosoni A, Blatt V, Pession A, Tallini G, Bertorelle R, Bartolini S, Calbucci F, Andreoli A, Frezza G, Leonardi M, Spagnolli F and Ermani M: MGMT promoter methylation status can predict the incidence and outcome of pseudoprogression after concomitant radiochemotherapy in newly diagnosed glioblastoma patients. J Clin Oncol 26: 2192-2197, 2008.

16. Brandsma D, Stalpers L, Taal W, Sminia P and van den Bent MJ: Clinical features, mechanisms, and management of pseudoprogression in malignant gliomas. Lancet Oncol 9: 453-461, 2008. 
17. Lee SW, Fraass BA, Marsh LH, Herbort K, Gebarski SS, Martel MK, Radany EH, Lichter AS and Sandler HM: Patterns of failure following high-dose 3-D conformal radiotherapy for high-grade astrocytomas: A quantitative dosimetric study. Int J Radiat Oncol Biol Phys 43: 79-88, 1999.

18. Chan JL, Lee SW, Fraass BA, Normolle DP, Greenberg HS, Junck LR, Gebarski SS and Sandler HM: Survival and failure patterns of high-grade gliomas after three-dimensional conformal radiotherapy. J Clin Oncol 20: 1635-1642, 2002.

19. Andersen AP: Postoperative irradiation of glioblastomas. Results in a randomized series. Acta Radiol Oncol Radiat Phys Biol 17: 475-484, 1978

20. Walker MD, Alexander E Jr, Hunt WE, MacCarty CS, et al: Evaluation of BCNU and/or radiotherapy in the treatment of anaplastic gliomas. A cooperative clinical trial. J Neurosurg 49 333-343, 1978.

21. Shapiro WR and Young DF: Treatment of malignant glioma A controlled study of chemotherapy and irradiation. Arch Neurol 33: 494-500, 1976.

22. Wallner KE, Galicich JH, Krol G, Arbit E and Malkin MG: Patterns of failure following treatment for glioblastoma multiforme and anaplastic astrocytoma. Int J Radiat Oncol Biol Phys 16: 1405-1409, 1989.
23. Shapiro WR, Green SB, Burger PC, et al: Randomized trial of three chemotherapy regimens and two radiotherapy regimens and two radiotherapy regimens in postoperative treatment of malignant glioma. Brain Tumor Cooperative Group Trial 8001 J Neurosurg 71: 1-9, 1989 .

24. Milano MT, Okunieff P, Donatello RS, Mohile NA, Sul J, Walter KA and Korones DN: Patterns and timing of recurrence after temozolomide-based chemoradiation for glioblastoma. Int J Radiat Oncol Biol Phys 78: 1147-1155, 2010.

25. McDonald MW, Shu H-KG, Curran WJ and Crocker IR: Pattern of failure after limited margin radiotherapy and temozolomide for glioblastoma. Int J Radiat Oncol Biol Phys 79:130-136, 2011

26. Sherriff J, Tamangani J, Senthil L, Cruickshank G, Spooner D, Jones B, Brookes C and Sanghera P: Patterns of relapse in glioblastoma multiforme following concomitant chemoradiotherapy with temozolomide. Br J Radiol 86: 20120414, 2013. 